\title{
Effect of Transformational Leadership on Nursing Informatics Competency of Chinese Nurses: The Intermediary Function of Innovation Self-Efficacy
}

\author{
Jie JING, Eksiri NIYOMSILP, Linli XIE, Haixia JIANG AND RONG LI
}

\begin{abstract}
Nursing informatics competency has crucial functions in the development of a hospital and patient safety. On the basis of the theory of selfconcept, there has been an inadequate study of the influence of transformational leadership on nursing informatics competency mediated by innovation selfefficacy. A descriptive correlational research design was conducted for 586 Chinese nurses from five public hospitals. The measurements were obtained from a questionnaire on transformational leadership, nursing informatics competency, innovation self-efficacy, and a sociodemographic datasheet. We identified a positive correlation between transformational leadership and nursing informatics competency. Innovation self- efficacy had a mediating effect. Managers need to provide measures to shape their transformational leadership style and promote innovation self-efficacy of nurses, which enhances nursing informatics competency of nurses.
\end{abstract}

Keywords. Transformational leadership, nursing informatics competency, innovation self- efficacy, nurse, China

\section{Introduction}

In 2007, the Quality and Safety Education for Nursing (QSEN) program in the United States proposed that nursing informatics competencies were divided into knowledge, skills, and attitude components [1]. Previous studies have shown that individual factors (such as trait, attitude, behavior, etc.) and situational factors (such as leadership style, organizational culture, organizational atmosphere, etc.) can independently or jointly affect employees' informatics competency [2]. Numerous empirical studies in the West and China have demonstrated the leadership of transformational leader's effectiveness [3]. Innovation Self-efficacy is a crucial component of employee self-concept, which is an individual's confidence that he or she can use the skills he or she possesses to solve problems creatively in a particular field and achieve innovative results [4]. Based on self- concept theory, the head nurses with transformational leadership style send their respect and recognition signals to nurses through incentive feedback, which may enable nurses to produce innovative self-efficacy in the process of interaction with them, thus promoting nurses to better build nursing informatics competency.

In conclusion, a theoretical framework was established (Figure 1). 


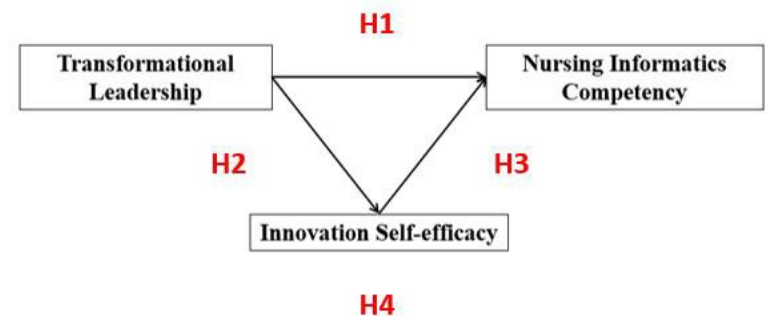

Figure 1. Theoretical framework of nursing informatics competency of nurses.

\section{Methods}

\subsection{Study Design and Sample}

We recruited 586 nurses from 5 public hospitals from November 2020 to February 2021. We recruited 140 participants in each hospital, and we handed out 700 questionnaires. Among them, 586 were valid questionnaires, so the effective sample rate was $83.71 \%$.

\subsection{Variables and Measurements}

\subsubsection{Demographic Information Data}

The demographic information consisted of seven variables, including gender, date of birth, marital status, education, professional level, date of starting work, and work department.

\subsubsection{Measures}

All measurement scales were proven to have good reliability and validity. The rating of each item was on a five-point Likert-type scale, from strongly disagree $=1$, disagree $=$ 2 , neutral $=3$, agree $=4$, to strongly agree $=5$.

\subsubsection{Transformational Leadership}

We used the version of the transformational leadership scale proposed by Chaoping Li \& Kan Shi. The dimensions of morale modeling, vision stimulation, individualized consideration, and charisma or idealized influence were $0.94,0.93,0.94$, and 0.94 .

\subsubsection{Nursing Informatics Competency}

The nursing informatics competency scale established by Luo et al. (2020) was conducted in this study. This scale had an overall Cronbach's coefficient of 0.947 .

\subsubsection{Innovation Self-efficacy}

We used the innovation self-efficacy scale developed by Tierney \& Farmer to measure innovation self-efficacy, which contains eight items. The reliability was 0.946 .

\subsection{Data Analysis}

SPSS Statistics 26.0 and SPSS Amos 24.0 for Windows were used for data coding, cleaning, and analysis. 


\section{Ethical Approval}

Ethical conformity approval was obtained from the Ethics Board at Sichuan Academy of Medical Science \& Sichuan Provincial People's Hospital (No.2020-90, issued on January 20, 2020).

\section{Results}

\subsection{Demographic Data}

The participants generally are as follows: mainly female $(\mathrm{N}=550,93.9 \%)$, most were over 25 years old $(\mathrm{N}=538,91.8 \%)$, most had a bachelor's degree $(\mathrm{N}=448,76.5 . \%)$, most were primary nurses $(\mathrm{N}=372,63.5 \%)$, and most of the participants $(\mathrm{N}=463$, $79.0 \%$ ) had more than 6 years experience in nursing. Descriptive statistics are shown in Table 1.

Table 1. Descriptive analysis.

\begin{tabular}{llll}
\hline Variables & Average score range & Mean & $S D$ \\
\hline Nursing Informatics Competency & & 4.01 & 0.78 \\
Nursing Information Consciousness & $1-5$ & 4.00 & 0.84 \\
Computer 0peration Competency & $1-5$ & 4.05 & 0.81 \\
Computer Software Management Competency & $1-5$ & 3.93 & 0.80 \\
Nursing Information Operation Competency & $1-5$ & 3.99 & 0.81 \\
Nursing Information Management Competency & $1-5$ & 4.03 & 0.78 \\
Innovation Self-efficacy & & 4.09 & 0.78 \\
Transformational Leadership & & 4.03 & 0.83 \\
Morale Modeling & $1-5$ & 3.94 & 0.85 \\
Vision Stimulation & $1-5$ & 4.04 & 0.83 \\
Individualized Consideration & $1-5$ & 4.06 & 0.82 \\
Charisma or Idealized Influence & $1-5$ & 4.09 & 0.86 \\
\hline
\end{tabular}

Abbreviation: $S D$, standard deviations.

\subsection{Correlation Study}

The correlation was measured according to Pearson' test (see Table 2).

Table 2. The correlations of variable sin the study.

\begin{tabular}{llll}
\hline Variables & 1 & 2 & 3 \\
\hline Nursing Informatics Competency & 1 & & \\
Innovation Self-efficacy & $0.994 * *$ & 1 & 1 \\
Transformational Leadership & $0.969 * *$ & $0.960 * *$ & 1 \\
\hline
\end{tabular}

$* * p<0.01$. 


\subsection{Regression Analysis}

In order to explore the influence of transformational leadership on nursing informatics competency, this study uses multiple linear regression, the results are shown in Table 3.

Table 3. Regression analysis.

\begin{tabular}{lccccc}
\hline & \multicolumn{2}{c}{$\begin{array}{c}\text { Unstandardized } \\
\text { Coefficients }\end{array}$} & Standardized & & Sig. $(t)$ \\
& $\mathrm{B}$ & Std. Error & $\beta$ & & \\
\hline Constant) & 0.250 & 0.039 & & 6.456 & $<0.001$ \\
Morale Modeling & -0.004 & 0.039 & -0.004 & -0.098 & 0.922 \\
Vision Stimulation & 0.218 & 0.037 & 0.230 & 5.844 & $<0.001$ \\
Individualized Consideration & 0.423 & 0.038 & 0.443 & 11.128 & $<0.001$ \\
Charisma or Idealized Influence & 0.288 & 0.047 & 0.316 & 6.169 & $<0.001$ \\
\hline \multicolumn{1}{c}{ Adjusted R ${ }^{2}$} & & 0.946 & Durbin-Watson & & 2.018 \\
\multicolumn{1}{c}{$F$} & & 2567.061 & Sig. $(F)$ & $<0.001$ \\
\hline
\end{tabular}

\subsection{The Structural Equation Models}

According to the theoretical framework, we establish a structural equation model, including two latent variables and ten measured variables (Figure 2). Results show that the model was fitted to the data (see Table 4).

\subsection{Direct and Indirect Effects}

We check the direct and indirect effects of innovation self-efficacy, shown in Table 5.

Table 4. Model fitting index.

\begin{tabular}{lllllllllllll} 
& \multicolumn{3}{c}{ Absolute adaptation index } & \multicolumn{3}{c}{ Value-added adaptation index } & \multicolumn{3}{c}{ Parsimony adaptation index } \\
\hline $\begin{array}{lllllllllllll}\text { Test statistic RMSEA } & \text { GFI } & \text { AGFI } & \text { NFI } & \text { RFI } & \text { IFI } & \text { TLI } & \text { CFI } & \text { PGFI } & \text { PNFI } & \text { PCFI } \\
\begin{array}{l}\text { Adaptation } \\
\text { standard }\end{array} & <0.1 & >0.8 & >0.8 & >0.8 & >0.8 & >0.8 & >0.8 & >0.8 & >0.5 & >0.5 & >0.5 \\
\begin{array}{l}\text { Data } \\
\text { efficacy }\end{array} & 0.094 & 0.804 & 0.779 & 0.950 & 0.932 & 0.952 & 0.934 & 0.952 & 0.583 & 0.697 & 0.698\end{array}$ \\
\hline
\end{tabular}

Abbreviations: AGFI, adjusted goodness of fit index; CFI, comparative fit index; GFI, goodness of fit index; IFI, incremental fit index; NFI, normed fit index; PCFI, parsimony comparative fit index; PGFI, parsimony goodness fit index; PNFI, parsimony normed fit index; RESEA, root mean square error of approximation; RFI, relative fit index; TLI, Tucker- 


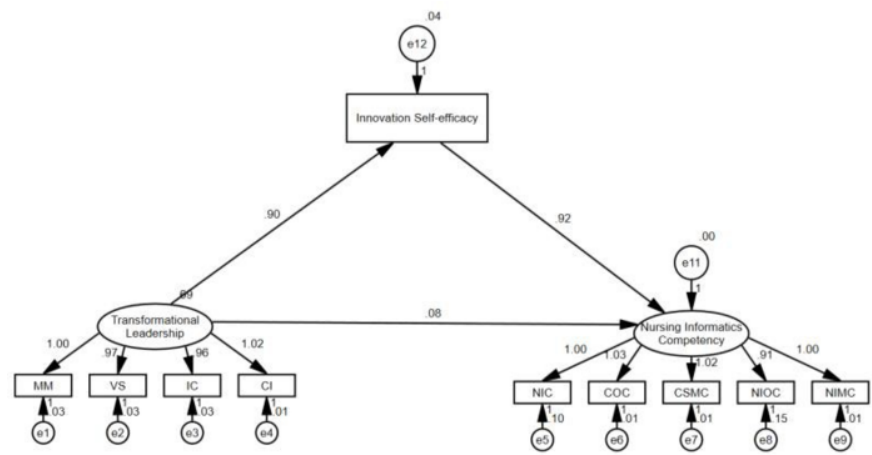

Figure 2. Structural equation model of nursing informatics competency of nurses.

Table 5. Direct and indirect effects.

\begin{tabular}{|c|c|c|c|c|c|}
\hline \multirow[b]{2}{*}{ Effect } & \multicolumn{3}{|c|}{ Product of coefficients } & \multicolumn{2}{|c|}{$\begin{array}{c}\text { Bootstrapping } 95 \% \\
\text { BC Confidence Interval (CI }\end{array}$} \\
\hline & Point estimate & Boot $S E$ & $Z$ (Sig.) & Lower & Upper \\
\hline \multicolumn{6}{|l|}{ Total effects } \\
\hline $\begin{array}{l}\text { Transformational Leadership on Nursing } \\
\text { Informatics Competency }\end{array}$ & 0.907 & 0.016 & $56.69(0.005)$ & 0.871 & 0.940 \\
\hline Direct effects & & & & & \\
\hline $\begin{array}{l}\text { Transformational Leadership } \rightarrow \text { Nursing } \\
\text { Informatics Competency }\end{array}$ & 0.075 & 0.025 & $3(0.014)$ & 0.028 & 0.122 \\
\hline \multicolumn{6}{|l|}{ Indirect effects } \\
\hline $\begin{array}{l}\text { Transformational } \\
\text { Leadership } \rightarrow \text { Innovation Self- } \\
\text { efficacy } \rightarrow \text { Nursing Informatics }\end{array}$ & 0.832 & 0.025 & $33.28(0.011)$ & 0.781 & 0.875 \\
\hline
\end{tabular}

Competency

\section{Conclusion}

In this study, we describe the situation of nursing informatics competency of Chinese nurses in public hospitals quantitatively. At the same time, we tested the correlation between variables. Also, we verified the mechanism of transformational leadership on nursing informatics competency and confirmed the mediating function of innovation self-efficacy.

\section{References}

[1] Hussey PA, Kennedy MA. (2016). Instantiating informatics in nursing practice for integrated patient centred holistic models of care: A discussion paper. Journal of advanced nursing, 72(5), 1030-1041.

[2] Hart MD. (2008). Informatics competency and development within the US nursing population workforce: A systematic literature review. CIN: Computers, Informatics, Nursing, 26(6), 320-329.

[3] Li Y, Castaño G, Li Y. (2018). Linking leadership styles to work engagement. Chinese Management Studies.

[4] Park NK, Jang W, Thomas EL, Smith J. (2020). How to organize creative and innovative teams: Creative self-efficacy and innovative team performance. Creativity Research Journal, 1-12. 